\title{
Die Zukunft kehrt zurück:
}

\author{
Die katholische Presse in der Gegenwart (I)
}

\author{
von $K$. Rüdiger Durth
}

\section{Es gab Publik}

„Es gibt keinen Index mehr / und auch das Imprimatur ist der Zeit angepaßt / Hexen werden schon lange nicht mehr verbrannt / und Syllabus und Antimodemisteneid gehören - zumindest offiziell - der Vergangenheit an / Der Papst spendet seinen Segen und Elektronik übermittelt ihn in alle Welt / Es gibt Radioprediger und Fernsehpfarrer / Welttage der Kommunikationsmittel / kirchliche Journalistenpreise und eine Pastoralinstruktion / Es gibt eine Mediendienstleistungsgesellchaft mbH und zwei zusätzliche Herausgeber beim Rheinischen Merkur / Es gab Publik.”

In diese Verse hat Harald E. Gersfeld seine „Bestandsaufnahme” der katholischen Presse der Gegenwart gefaßt ${ }^{1}$, eine düstere Prognose, die „das Ende der katholischen Presse”, von Hans Wagner Mitte der 70 er Jahre dreibändig publikumswirksam eingeläutet ${ }^{2}$, verheißt. Im Gegensatz dazu stehen hoffnungsvolle Prognosen der Arbeitsgemeinschaft Katholische Presse (AKP) auf der 29. Jahresversamlung im Oktober $1978^{3}$, große Werbekampagnen, die mit dem 85 . Deutschen Katholikentag in Freiburg eingeläutet wurden ${ }^{4}-$ aber es gilt auch die nachdrückliche Mahnung des Mitherausgebers des „Rheinischen Merkur”, Hans Maier: „Das Verhältnis der deutschen Katholiken zu ihrer eigenen Presse bedarf einer Korrektur. Die negativen Auflagenentwicklungen der meisten katholischen Presseorgane zeigen, daß in weiten Teilen des Katholizismus der Wert und die Notwendigkeit einer gesunden katholischen Presse nicht erkannt ist. Wir sollten alles daransetzen, das Feld der Meinungsbildung nicht nur anderen zu überlassen."

Was stimmt? Im zurückliegenden Jahrzehnt hat die katholische Kirche große, meist von der Öffentlichkeit kaum bemerkte Anstrengungen unternommen, um die in eine große Krise geratene katholische Presse zu retten. Die mit erheblichen finanziellen Mitteln betriebene Bestands- und Sanierungsüberprüfung beginnt zu greifen. Die Auflagen stabilisieren sich $^{6}$, die Verantwortlichen fassen neuen Mut. Die Zukunft der katholischen Presse kehrt zurück, auch wenn noch ein langer Weg zu beschreiten ist.

Es gab „Publik” - für viele noch immer ein schmerzlicher Punkt in der Nachkriegsgeschichte katholischer Publizistik. In einem Brief an Journalisten schreibt der Herausgeber des „Publik-Forum”, Heinz-Wilhelm Brockmann, im September 1978: „Wir meinen, daß die katholische Medienlandschaft seit dem Ende von 'Publik' am 15. November 1971 einseitig ausgerichtet ist. Wenn jetzt eine andere Zeitung, der 'Rheinische Merkur' - nicht zuletzt durch die 'Salbung' offizieller Herausgeber - eine Art Organ amtlicher katholischer Stellen werden soll, dann wird diese Tendenz noch verstärkt. Den 'runden Tisch' für alle Katholiken, an dem offen und sachbezogen diskutiert wird, den gibt es unseres Erachtens seit 1971 nicht mehr."7

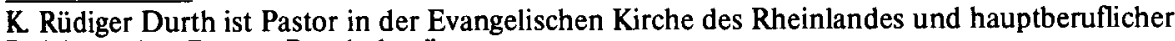
Redakteur der „Bonner Rundschau”. 
Für den Leiter der Zentralstelle Medien im Sekretariat der Deutschen Bischofskonferenz, Prälat Wilhelm Schätzler, freilich wird der „Publik-Schock überbewertet. Die Einstellung war ein rein verlagswirtschaftliches Problem, kein ideologisches. "8 Während die 1969 gegründete und knapp drei Jahre später wieder eingestellte Wochenzeitung „Publik”, die insgesamt 25 Millionen DM Subvention aus Kirchensteuermitteln verschlang', für die offizielle kirchliche Medienpolitik keine Rolle mehr spielt, wird das Thema nicht zuletzt von der Leserinitiative Publik e.V. wachgehalten, die sich als „Erbe” versteht und seit numehr acht Jahren das „Publik-Forum” herausgibt - mit 13.000 Abonnenten, „die eindeutig 'opinion-leader-Qualität' hat" 10 .

Die Medienpolitik der katholischen Kirche hat sich hingegen der Stärkung der 22 Bistumszeitungen, der Sicherung von „Rheinischer Merkur" und „weltbild" sowie anderer wichtiger Zeitschriften und Verlagsobjekte und dem Ausbau der Katholischen Nachrichtenagentur (KNA) sowie der Förderung desjournalistischen Nachwuchses zugewandt.

\section{Der Merkur-Streit}

Verfügte die katholische Kirche vor 1933 über rund 400 Tageszeitungen, die 10 Prozent dergesamten Auflage der deutschen Tagespresse ausmachten ${ }^{11}$, so entwickelte sich nach 1945 die katholische Publizistik zur Zeitschriftenpresse ${ }^{12}$. Die einzigen bedeutenden Ausnahmen bildeten die Wochenzeitung "Rheinischer Merkur" und die dreimal in Würzburg erscheinende „Deutsche Tagespost”. Beide Zeitungen gelten als CDU-nah bis rech tskonservativ.

Die "Deutsche Tagespost" mit einer gegenwärtigen Auflage von 33.000 Exemplaren ${ }^{13}$ konnte im Spätsommer 1978 ihr 30jähriges Bestehen feiern. Ihr Verbreitungsgebiet umfaßt hauptsächlich den süddeutschen Raum. CSU-Vorsitzender Franz Josef Strauß schrieb der „Deutschen Tagespost” ins Jubiläums-Stammbuch: „Während große Teile der deutschen Publizistik, vor allem in auflagenstarken Massenmedien oder in den öffentlich-rechtlichen Rundfunkanstalten, dem verhängnisvollen Irrtum anhingen, da $B$ 'links' die einzig tragbare politische Mode sei, hat die Redaktion der 'Deutschen Tagespost' allzeit klaren Kopf be- und klaren Kurs gehalten." ${ }^{14}$ Oder Rottenburgs Bischof Georg Moser, Vorsitzender der Publizistischen Kommission der Deutschen Bischofskonferenz: „Im pluralistischen Konzert einer nur noch wenig bekenntnisfreudigen Presse erhebt sie (die 'Deutsche Tagespost') vernehmlich und entschieden ihre katholische Stimme, und für eine engagierte Meinungsgruppe im deutschen Katholizismus hat sie sich als unverwechselbares publizistisches Organ konsolidiert." ${ }^{15}$

An dieser Tageszeitung entzündet sich bundesweit kaum Streit, zumal sie sich auch finanziell selbst trägt. Anders hingegen beim ${ }_{n}$ Rheinischen Merkur", dessen Fusionierung 1970 mit „Publik” scheiterte. Die konservative Wochenzeitung verlor ständig an Auflage und geriet dadurch in eine große Gefahrenzone. Geschockt von „Publik” und den negativen Folgen in der Öffentlichkeit entschlossen sich 1974 sieben Bistümer, allen voran das Erzbistum Köln, die Mehrheitsanteile dieser Zeitschrift zu erwerben. Acht Millionen DM sollen es gewesen sein. Der Herausgeber von „publik-Forum”, Heinz Wilhelm Brockmann, schätzt, daß der „Rheinische Merkur” bislang rund 18 Millionen DM an Subventionen verschlungen hat und fortan jährlich zwei Millionen ZuschuB erhält ${ }^{16}$. Bestätigungen für diese Zahlen sind nicht zu erhalten, da sie von den Beteiligten als „vertraulich” behandelt werden. 
Im Frühjahr 1978.wurde die bisherige Herausgeberschaft von Otto B. Roegele um den Präsidenten des Zentralkomitees der deutschen Katholiken (ZdK), Hans Maier, und die evangelische Publizistin Christa Meves erweitert. Dieser Schritt sollte zugleich eine inhaltlicheÖffnung derZeitung bewirken, neue Leserkreise erschließen und auch stärker in die evangelische Kirche hineinwirken. Gleichzeitig wurde ein redaktionelles Ausbauprogramm beschlossen und zum 85. Deutschen Katholikentag in Freiburg 1978 eine breitangelegte Werbekampagne gestartet ${ }^{17}$. Mittelfristiges Ziel ist es, die Auflage auf 100.000 Exemplare pro Woche zu steigern (bislang rund 60.000 Exemplare) ${ }^{18}$.

Wird damit versucht, den „Rheinischen Merkur”, zur offiziellen katholischen Wochenzeitung zu erheben? Das „Publik-Forum” fürchtet dies, stellt aber klar: „Der' Rheinische Merkur' ist nicht die Wochenzeitung $d$ e $r$ deutschen Katholiken und sie kann es auch nicht werden." ${ }^{19}$ Der Geschäftsführer der 1975 gegründeten Mediendienstleistungsgesellschaft (MDG), Raimund Brehm, antwortete auf die Attacken von „Publik-Forum”:

„Es ist richtig, daß die Zeit vorbei ist, in der sich die katholische Kirche ein Sprachrohr leistet, das insgesamt weit mehr als 25 Millionen DM Subvention kostet und bei seiner Einstellung eine unstabile verkaufte Auflage von wenig mehr als 70.000 Exemplaren aufweist. Es bleibt zu hoffen, daß diese Größenordnungen für die Förderung eines einzigen Objekts nicht wiederkehren, da man sonst zu Recht den deutschen Bischöfen ein einseitiges Medienkonzept vorwerfen würde. Das jetzt praktizierte Konzept aber ist unserer Meinung nach umfassend und pluralistisch angelegt. In seinem Rahmen ist der'Rheinische Merkur', um den es Ihnen ja geht, nur ein Objekt unter vielen." ${ }^{20}$ Innerhalb der katholischen Kirche wird die Stützungsaktion für den „Rheinischen Merkur” nach wie vor mit Argwohn beobachtet, auch wenn der Hohn des sozialdemokratischen „Vorwärts" nicht geteilt wird, es handele sich um ,katholische Kosmetik über alten Falten" 21 . Man geht davon aus, daß der Kirche keine andere Wahl blieb, um nicht auch diese Zeitung noch einstellen zu müssen, und auch davon, $\mathrm{da} ß$ es der erweiterten Redaktion gelingt, das schlechte Image abzubauen. Prälat Wilhelm Schätzler:„Das Blatt ist besser als sein Ruf." 22

\section{Stille Kraftakte}

Ende der60er Jahre setzte für die katholische Kirche in der Bundesrepublik Deutschland eine grundlegende Wende ein, die wesentiche Auswirkungen auch auf ihre Presse hatte, die man bis zu diesem Zeitpunkt mehr oder weniger als selbstverständlich hingenommen hatte. Zumal sich die Kirche durch eine weitgehende Feindlichkeit oder Abneigung gegenüber allem, was mit Journalismus zu tun hatte, auszeichnete.

Erstmals schlug die längst in Gang gekommene Säkularisierung der Gesellschaft voll durch. Die Teilnahme an der Messe sank rapide $a^{23}$, die kirchliche Bindung schwand und damit auch die Zahl der Abonnenten von Bistumszeitungen, die in einem direkten Verhältnis zur Kirchlichkeit steht ${ }^{24}$. Die Auflage sank von weit über zwei Millionen Exemplaren pro Woche erstmals unter die Zwei-Millionen-Grenze ${ }^{25}$.

Gleichzeitig vollzog sich in Bonn der Regierungswechsel von CDU/CSU zur sozialliberalen Koalition mit zahlreichen Folgen für das überkommene Gesellschaftsbild der katholischen Kirche. Für die Massenmedien wurde die Berichterstattung über die Kirche uninteressanter, hingegen unterstützten sie weithin Tendenzen, die der katholischen Kirche zuwiderliefen. Hier sei nur an den Streit um die Reform des Abteibungsparagraphen 218 Strafgesetzbuch, die Grundwertediskussion, die Reformen des Ehe- und 
Scheidungs- sowie des Strafrechts beispielhaft erinnert. Im allgemeinen Trend „weg von der Kirche" erinnerte man sich wieder der eigenen Presse, die so plötzlich selbst in größte Schwierigkeiten geraten war.

Parallel dazu wirkte sich - medienpolitisch gesehen - das II. Vatikanische Konzil aus, das auch im Blick auf die sozialen Kommunikationsmittel neue Wege gezeigt hatte, die ihren formalen Abschluß in der Pastoralinstruktion „Communio et progressio" 1971 fanden ${ }^{26}$. In diesen Zeitraum fällt dann auch der sogenannte „Publik-Schock”.

Nun entschloß sich die katholische Kirche zu einem bislang kaum für möglich geahnten Kraftakt, dessen pressepolitische Auswirkungen erst heute auch öffentlich registriert werden und zur Hoffnung auf die Rückkehr der Zukunft für die katholische Presse allen Anlaß geben.

Neben den bereits erwähnten Stützungsaktionen für den „Rheinischen Merkur” und die Augsburger Illustrierte "Weltbild" -wurden folgende wichtige Maßnahmen ergriffen: Gründung des „Instituts zur Förderung publizistischen Nachwuchses e.V.” in München, eine breitangelegte Feldbefragung der deutschen Katholiken über ihr Verhältnis zur Bistumspresse ${ }^{27} 1975$, im gleichen Jahr die wohl wichtigste Einrichtung der Mediendienstleistungsgesellchaft (MDG) München, die Stärkung der Zentralstelle Medien, neue Aktivitäten der 1948 gegründeten Arbeitsgemeinschaft Katholische Presse (AKP), Ausbau der Bistumspresse und insgesamt ein größeres, offeneres Verhältnis gegenüber den Journalisten. Dazu einige Erklärungen von Joseph Kardinal Höffner, dem Vorsitzenden der Deutschen Bischofskonferenz:

In einem Grußwort zum 25jährigen Bestehen der KNA: „Das Verhältnis der Kirche zur Publizistik war und ist vielfach mit falschen Vorstellungen, auch mit Emotionen belastet ... Seit dem Zweiten Vatikanischen Konzil hat sich die Kirche um ein neues Verhältnis zu den Medien bemüht. Dieses ernste Bemühen war nicht erfolglos . . . Die Kirche braucht die Medien, um mit der Gesellschaft ins Gespräch zu kommen. Sie ist darauf angewiesen, daß die in den Medien Tätigen bereit sind, sachlich und fair über die Fragen der Kirche zu berichten. ihr Verhältnis zu den Medien ist nicht spannungsfrei, aber es ist auch nicht grundsätzlich gestört." 28

Zum 30jährigen Bestehen der „Deutschen Tagespost”: „Wer als katholischer Publizist die Wirklichkeit der Kirche darstellen will, kann sich Mißverständnissen nicht entziehen ... Man sollte solche Mißverständnisse von beiden Seitennicht dramatisieren. Es ist erfreulich, daß in den letzten Jahren ... das Verhältnis der Kirche zu den Medien - aber auch umgekehrt -'normaler' geworden ist. Beide Seiten haben dazu ihren Beitrag geleistet. ${ }^{29}$

Als Erfolg hat sich auch die 1970 gegründete Konpress-Anzeigen e.G. erwiesen, in der sich 24 katholische und 8 evangelische Wochenblätter zu einem Anzeigenring zusammengeschlossen habe. Das Jahr 1977 konnte Konpress mit einem Plus von 24,5 Prozent des Nettoumsatzes (entspricht rund 1,1 Millionen DM) gegenüber 1976 abschließen. Allerdings bezog sich die Steigerung ausschließlich auf die Ausweitung des Beilagengeschäftes. Die diesem Anzeigenring angeschlossenen kirchlichen Blätter erreichen 12,3 Prozent derGesamtbevölkerung ab 14 Jahren (5,74 Millionen Menschen) ${ }^{30}$. Bereits 1971 hatte Konpress eine Leseranalyse durchgeführt ${ }^{31}$. 


\section{MDG - „eine Art Kienbaum-Beratung"}

$\mathrm{Zu}$ den wichtigsten Neuerungen auf dem katholischen Medienmarkt des letzten Jahrzehnts zählt die 1975 miteinem Stammkapital von 150.000 DMgegründete Mediendienstleistungsgesellschaft (MDG) mit Sitz in München - für Prälat Wilhelm Schätzler "eine Art Kienbaum-Beratung, doch viel mehr".

Die MDG ist ein Teil des „Publizistischen Sofortprogramms”, das 1972 auf der Gemeinsamen Bistumssynode bekanntgegeben wurde. Ziel des Sofortprogramms war es ja, eine Einrichtung zu schaffen, die die Vielfalt der katholischen Presse sichern half. Gesellschafter der MDG ist der Verband der Diözesen. Großer Wert wird auf das Wort Dienst gelegt, um von vornherein sicherzustellen, $\mathrm{da} B$ es sich bei dieser Gesellschaft nicht um ein Steuerungssystem handelt. Aufgabe ist es vielmehr, allen katholischen Zeitungs-, Zeitschriften- und Buchverlagen fachliche Hilfen zu gewähren.

Bischof Georg Moser, Aufsichtsratsvorsitzender der MDG: „Die Charakteristik der MDG liegt in der Förderung eines ganzheitlichen Medienkonzeptes bei gleichzeitiger Realisierung einer optimalen Entfaltung der Einzelmedien. Dem Gesamtkonzept liegt der Gedanke zugrunde, daß sich die unterschiedlichen Medien, auch die jeweiligen Einzeltitel, funktional ergänzen und somit nicht im Verdrängungswettbewerb untereinander konkurrieren, sondern im Zusammenwirken eine Kommunikationskette bilden. Die Schwierigkeiten, ein derart komplexes Medienprogramm aufeinander abzustimmen, ist dadurch zu lösen, daß man den gemeinsamen Nenner sucht." 32

Nach folgenden Prinzipien arbeitet die MDG, für die es bislang kein Gegenbeispiel gibt: Stabilisierung der jetzt vorhandenen katholischen Medien, marktgerechte Weiterentwicklung, Heranbildung eines fachlich qualifizierten Managements undjournalistischen Nachwuchses.

Moser: „Die MDG ist Ausdruck einer positiven Einstellung der Kirche zu den Medien, ihren Aufgaben und ihren Bedürfnissen. Wir haben erfahren, welche Bedeutung die Medien haben, wenn es darum geht, die Botschaft und Aktivitäten der Kirche in der Öffentlichkeit darzustellen. Deshalb ist die Arbeitsweise der MDG nicht allein auf das Halten eines status quo ausgerichtet ... Uns scheint, daß die MDG ein entwicklungsfähiges Service-Instrument, eine Chance für die Zukunft der katholisch-orientierten Medien ist. Was sie will, ist die wirtschaftliche und publizistische Stärkung des katholischen Medienbereichs und dadurch eine verstärkte pastorale Effizienz." ${ }^{33}$

Oberstes Arbeitsprinzip der MDG ist „erst Diagnose, dann Therapie”. Die wichtigsten Leistungen aus der Sicht von Geschäftsführer Raimund Brehm:

- 1976 Bestandsaufnahme bei den 64 ka tholischen Zeitungs-und Zeitschriftenverlagen, die der AKP angehören. Der 170seitige Berichtsband ist den Verlagen im Februar 1978 zugestellt worden.

- 1977 begann eine Bestandsaufnahme bei den 71 Buchverlagen und 178 Buchhandlungen des Verbandes Katholischer Verleger und Buchhändler. Der Bericht soll Ende 1978 vorgelegt werden.

- Untersuchung der Schriftenstände in den Kirchen und Entwicklung von Modellversuchen.

- Verstärkte Ausbildungsförderung von Redaktionsvolontären bei der katholischen Presse. 1978.wurden so über 20 Volontärplätze geschaffen. 
- Fortbildung des Verlags-Managements.

- Zusammen mit den Verlagen der Kirchenpresse werden Vertriebs- und Werbemaßnahmen entwickelt, die die Gewinnung neuer Leserschaften und die Verbesserung der Haltbarkeit vorhandener Leserschaften beinhaltet.

- Erarbeitung eines einheitlichen Grundwerbemittels für die gesamte Kirchenpresse.

- Erforschung der Marktchancen einer katholisch orientierten Wochenzeitung - im Blick auf den „Rheinischen Merkur”.

- Gemeinschaftswerbung für das katholische Buch.

- Seit 1977 ist die MDG Hauptgesellchafter der Katholischen Filmwerk GmbH in Frankfurt ${ }^{34}$.

Raimund Brehm: „Es genügt heute nicht mehr, mit kirchlichen Empfehlungen im Lesermarkt zu operieren und an die Solidarität des Kirchenvolks zu appellieren ... Endziel einer lesergerechten 'Öffnung nach außen' müßte wohl sein, daß sich katholische Zeitschriften auch im freien Leistungswettbewerb erfolgreich messen können, daß sie am Kiosk ebenso ihre reelle Chance suchen." ${ }^{35}$

\section{AKP - der selbstlose Helfer}

Die Arbeitsgemeinschaft Katholische Presse (AKP) mit Sitz in St. Augustin bei Bonn zählt zu den großen selbstlosen Helfern innerhalb der katholischen Publizistik, und sie ist zugleich ein Beweis für die Leistungsfähigkeit publizistischer „Selbstverwaltung”. Auf die AKP gehen viele Initiativen zurück, ohne die die Krise der katholischen Presse Ende der 60er Jahre erheblich schärfer ausgefallen und ohne die die Lösung der anstehenden Aufgaben kaum zu meistern wäre. Ihre Zusammenarbeit mit der Zentralstelle für Medien, der Mediendienstleistungsgesellschaft und der Publizistischen Kommission ist eng, aber der von der kirchlichen Behörde unabhängige Status konnte erhalten werden.

Gegründet wurde die Arbeitsgemeinschaft (zunächst nannte sie sich Arbeitsgemeinschaft Kirchliche Presse, seit 1970 Katholische Presse) nach dem Zweiten Weltkrieg als freiwilliger und selbständiger Zusammenschluß katholischer Presseunternehmen. Heute sind ihr 65 Verlage und Unternehmungen mit 110 Wochenzeitungen und Zeitschriften angeschlossen, die eine Auflage von rund 11 Millionen Exemplaren repräsentieren und somit zu den größten Pressegruppen in der Bundesrepublik Deutschland zählen.

Finanziert wird die AKP aus den Beiträgen der Mitgliedsverlage. Jedoch ist die Zusammenarbeit von Verlegern und Redakteuren gleichberechtigt. Vorsitzender ist seit vielen Jahren der Chefredakteur der Kirchenzeitung Aachen, Dr. Ferdinand Oertel. Ferner arbeitet die AKP in der „Fachgruppe Konfessionelle Presse” des Verbandes Deutscher Zeitschriftenverleger (VDZ) und in der Katholischen Weltunion der Presse (UCIP) mit.

Geschäftsführer Bruno Geuter: „Die vielfältige Zusammen- und Mitarbeit ist für die Arbeitsgemeinschaft eine Voraussetzung, um die Belange der katholischen Kirchenpresse nach außen und in der Kirche wirksam vertreten zu können. Sie hat sich in den letzten Jahren darum bemüht, das Selbstverständnis der Kirchenpresse und ihres Auftrages in der nachkonziliaren Kirche zu formulieren und durch Öffentlichkeitsarbeit im außer- und innerkirchlichen Raum ein sachgerechtes Bild dieser Presse und ihrer Funktionen zu vermitteln." 
1972 legte die AKP die „Grundsätze zu einem Rahmenplan der Kirchenpresse” vor, beteiligte sich 1975 aktiv an der Felduntersuchung über die Kirchenpresse. Die Förderung und der Ausbau von Kooperationen zählt zu den Schwerpunktthemen der AKP. Der Erfolg:

Viele Bistumszeitungen sind in eine enge Zusammenarbeit getreten. Sie reicht von regelmäßigen Redaktionskonferenzen auf regionaler Ebene bis zur gemeinsamen Erstellung ganzer Zeitschriftenteile. Zwei Bistumsblätter in Bayern verfügen bereits über einen gemeinsamen Kupfertiefdruck-Mantel. Auch im norddeutschen Raum erstellen zwei Bistumszeitungen den überdiözesanen Teil ihrer Blätter gemeinsam. Die Kirchenzeitungen der Diözesen Fulda und Limburg erscheinen seit 1972 im gleichen Verlag.

Bruno Geuter: „Die Möglichkeiten zu Konzentrationen sind in der katholischen Kirchenpresse jedoch sehr begrenzt, bei den Bistumsblättern aufgrund ihres bewußten Charakters als Organe der Ortskirche kaum gegeben. Statt dessen muß eine vermehrte Kooperation zur Steigerung der publizistischen und wirtschaftlichen Leistungsfähigkeit führen." 36

Zusammen mit der MDG führte die Arbeitsgemeinschaft Katholische Presse 1978 eine bundesweite Werbekampagne durch, in deren Mittelpunkt ein 20seitiger Faltprospekt steht, der einen Überblick über die katholische Presse gibt. Damit verbunden ist eine Plakataktion und ein Schreiben an alle Pfarrämter, in denen die Geistlichkeit gebeten wird, die Sache der katholischen Presse zu unterstützen.

In hoher Auflage wurde der Prospekt auch auf dem 85. Deutschen Katholikantag in Freiburg verteilt. Beigelegt war eine Bestellkarte - für Probeexemplare oder Festbezug einer oder mehrerer Zeitschriften. Der Rücklauf der Bestellkarten ist nach Bruno Geuter „Zufriedenstellend”. Bis zu 100 Karten kamen an einem Tag in der AKP-Geschäfsstelle in St. Augustin an.

\section{Hochwürdige Presse im Wandel}

Die Bistumszeitung, früher mehr eine hochwürdige Presse denn ein lesernahes Informationsinstrument, befindet sich seit vielen Jahren in einem grundlegenden Wandel. Die meisten der 22 Bistumsblätter haben, wie das Zentralkomitee der deutschen Katholiken in einer von der Vollversammlung im Frühjahr 1978 verabschiedeten „Erklärung zur Situation der Bistumspresse" feststellt, , in zunehmendem Maße den Abschied von einer überwiegend erbaulichen und reproduktiven Selbstdarstellung des kirchlichen Lebens vollzogen"37.

Im Deutschlandfunk wurde am 21. April 1978 eine harte Kontroverse zwischen Dr. Hubert Hanisch und Dr. Ferdinand Oertel über die Bistumspresse ausgetragen, deren Positionen für die Auseinandersetzungen um diese wichtigste Säule der katholischen Publizistik beispielhaft stehen ${ }^{38}$.

Für Hanisch hält man das Blatt, „aber man hält nicht viel davon”. Die Aufspaltung in 22 Bistumsblätter trage die Hauptschuld für die nicht ausreichende Fortentwicklung dieser Presse. Hanisch in dieser Sendung: „Wo bleiben qualifizierte Auseinandersetzungen in Politik und Wirtschaft, Kultur und Gesellschaft?" Er fordert Abhilfe durch die 
Bischöfe und Schaffung einer Einheitszeitung für alle Bistümer: „Eine Hauptredaktion, das bedeutet mehr unterschiedliche Köpfe und Temperamente, mehr Meinungen, mehr Spezialisierungen der Interessen und Wissensgebiete, mehr Verschiedenheit des Alters, mehr Möglichkeiten für Reporter auf Reisen. Überfällig ist auch der Chefredakteur auf Lebenszeit . . . Ein Gesamtmantel könnte im gesamten deutsch sprechenden Gebiet ohne die Fehler der geistig diffusen und leicht größenwahnsinnigen 'Publik' zu wiederholen - eine Schicht von kritischen Katholiken ansprechen, die sich den kirchlich geförderten 'Rheinischen Merkur' - und sei es nur nach Vorurteil - niemals ins Haus holen würde ... Die.journalistische und pastorale Wirkung der Bistumspresse liegt weit unter der vielfach günstig erscheinenden Auflagenhöhe. Soll die Spendenmentalität abgebaut werden, bedarf es erheblicher Anstrengungen zur Verbesserung des Produktes."

Ferdinand Oertel konterte, daß die Bistumspresse sehr wohl gelesen werde, im Durchschnitt von 1,5 Lesern. Oertel: „Gelesen wird sie also doch, die Bistumspresse, und daß sie nur dem Pfarrer zuliebe oder um eine gute Sache finanziell zu unterstützen bezogen wird, auch das sind heute kaum noch Bezugsmotive."

Ergab zu, daß die Kirchenpresse seit langem unter einem schlechten Image leide, obwohl sie sich „weg vom alten Erbauungsblatt zu einem zeitgemäßen Informationsmittel” entwickelt hätte. Gerade im Zusammenhang mit „Publik” sei die Bistumspresse immer wieder als zweit- oer gar drittklassig abgestempelt worden - „und diese Behauptung hat sich bis heute gehalten".

Weiter führte Oertel in dieser Kontroverse aus: „Im Hinblick auf die Information im innerkirchlichen Raum muß man ohne Abstriche anerkennen, daß die Kirchenpresse in den vergangenen Jahren dieser Aufgabe nachgekommen ist. Gäbees sie nicht, wäre etwa über die Synode, über Entwicklungshilfe, über soziale Leistungen, über Grundwerte nicht soviel an Information und Hilfe zur Meinungsbildung ins Kirchenvolk gekommen, wie es der Fall ist." Oertel gab zu, daß die meisten Redaktionen von Kirchenzeitungen personell unterbesetzt seien, was geändert werden müsse, gleichzeitig wehrte er sich mit Nachdruck gegen eine Einheitskirchenzeitung, „die wieder am jetzigen Leserpublikum vorbei gemacht” sein würde. Denn „die Bistumsblätter (leben) vom Bistumsgeschehen. Sie sind Organe der Ortskirche, so wie das Konzil sie gezeichnet hat”. Außerdem verweist er auf die allgemeinen journalistischen Erfahrungen: „Wenn man zudem berücksichtigh, daß auch die allgemeine Presse immer stärker ihre Leserbindung durch orts- und lokalbezogene Ereignisse gewinnt, wäre es auch von daher verfehlt, die Bistumsblätter zusammenzulegen, weil dann notwendigerweise überregionale Themen in den Vordergrund rücken müßten."

Das Zentralkomitee der deutschen Katholiken verweist in seiner Erklärung auf die Feldbefragung 1975, nach der etwa die Hälfte der katholischen Gesamtbevölkerung die Kirchenpresse als Stimme der Kirche in der heutigen Informationsflut für wichtig oder besonders wichtig hält.

Die Konkretionen des Zentralkomitees im Blick auf die Bistumspresse ${ }^{39}$ :

- Rasche redaktionelle und verlegerische Anstrengungen, um die Bistumspresse „zu einem wirksameren Medium im innerkirchlichen und gesellchaftspolitischen Raum zu entwickeln".

- Stärkere Öffnung für die Probleme und Fragestellungen von Menschen, die der Kirche fernstehen. 
- Forum für ungehinderte und kritische Meinungsbildung innerhalb der Kirche.

- Weltoffenere Berichterstattung und zeitgemäße Sprache. Themen nicht auf innerkirchliche Probleme beschränken.

- Versuch des Vorstoßes in neue Lesergruppen, Gewinnung vor allem jüngerer Menschen.

- Junge Leser verlangen auch von der Bistumspresse „eine moderne, journalistischprofessionelle Darstellung der Inhalte”.

- Bevorzugte Berücksichtigung der Kirchenpresse bei der Informationsvermittlung durch die Amtskirche.

- „Die Bistumspresse als Medium der innerkirchlichen Kommunikation ist auf die Mitarbeit und die Unterstützung aller Katholiken des Bistums angewiesen. Die Leser tragen eine nicht geringe Mitverantwortung für das Gedeihen und die Wirkungsmöglichkeiten der kirchlichen Presse."

Wie sehr sich bereits die „Landschaft” geändert hat, verdeutlichen die „Überlegungen zur Aufgabe einer Bistumszeitung”, die der neue 35jährige Chefredakteur der Kirchenzeitung für das Erzbistum Köln, Dr. Hajo Goertz, zu seinem Amtsantritt im August 1978 veröffentlichte ${ }^{40}$ :

Die Kirchenzeitung ist keine „papierene Kanzel”, hat aber Verantwortung „für die Gestaltung von Kirche und Welt” und stellt ein „Forum für den innerkirchlichen Dialog” dar: „Der Pluralismus, die Vielstimmigkeit darf gerade in der Kirche nicht zur Polarisation, zur Unversöhnlichkeit führen; die Freiheit des Gesprächs muß in die Einheit der Partner münden. Die Kirchenzeitung als Instrument der sozialen Kommunikation hat da ein soziales Ziel, nämlich zum Verständnis, zur Verständigung untereinander beizutragen. Journalistisch: sie muß Ereignisse und Meinungen kommentieren, sie muß ordnen und werten. Insofern spiegelt sie den innerkirchlichen Dialog nicht nur wider, sie nimmt auch selbst an ihm teil."

Darüberhinaus findet Goertz, daß die Kirchenzeitung eine „kritische Funktion” und eine "Gesamtverantwortung für die Catholica” hat. Die Kirche der Dritten Welt müsse ebenfalls ihren Platz in der Kirchenzeitung haben - ,vielleicht muß die Redaktion dabei gelegentlich eine Zumutung für ihre Leser sein”. Außerdem komme der Bistumszeitung die Aufgabe zu, „hinein in die säkulare Welt” zu schauen.

\section{1,9 Millionen Auflage pro Woche}

22 Bistumszeitungen erscheinen wöchentlich - miteiner Auflage von rund 1,9 Millionen Exemplaren. Spitzenreiter ist nach wie vor „Kirche und Leben” in Münster mit rund 210.000 Exemplaren (und steigender Tendenz wieder) und die kleinste Kirchenzeitung das Berliner Petrusblatt mit 15.000 Auflage.

Seit dem großen Auflageneinbruch Ende der 60er Jahre hat sich zwischenzeitlich die Situation stabilisiert. Einige Bistumszeitungen melden ,steigende Tendenz”, andere „leichten Rückgang”. In der folgenden Tabelle geben wir einen Überblick über die Entwicklung der Bistumspresse zwischen Ende 1972 und Ende 1976, der bislang letzten exakten IVW-Zahlen (jeweils auf volle Tausend auf- oder abgerundet bei den Zahlen 1976). 


\begin{tabular}{llcc}
\hline Titel & Diözese & $\begin{array}{c}\text { Auflage } \\
\text { Ende 1972 }\end{array}$ & $\begin{array}{c}\text { Auflage } \\
\text { Ende 1976 }\end{array}$ \\
& & & \\
Kirchenzeitung & Aachen & 141.690 & 145.000 \\
Kirchenzeitung & Augsburg & 95.398 & 83.00 \\
St. Heinnchsblatt & Bamberg & 58.210 & 58.000 \\
Petrusblatt & Berlin & 15.336 & 15.000 \\
Kirchenzeitung & Eichstätt & 41.449 & 41.000 \\
Ruhrwort & Essen & 98.313 & 84.000 \\
Konradsblatt & Freiburg & 149.189 & 147.000 \\
Bonifatiusbote & Fulda & 20.779 & 19.000 \\
Kirchenzeitung & Hildesheim & 40.051 & 39.000 \\
Kirchenzeitung & Köln & 162.500 & 159.000 \\
Der Sonntag & Limburg & 36.369 & 33.000 \\
Glaube und Leben & Mainz & 46.780 & 46.000 \\
Kirchenzeitung & München & 110.476 & 96.000 \\
Kirche und Leben & Münster & 213.013 & 209.000 \\
Kirchenbote & Osnabrück & 61.793 & 59.000 \\
Der Dom & Paderborn & 139.475 & 131.000 \\
Bistumsblatt & Passau & 47.352 & 45.000 \\
Bistumsblatt & Regensburg & 90.173 & 86.000 \\
Kath. Sonntagsblatt & Rottenburg & 136.475 & 118.000 \\
Der Pilger & Speyer & 74.132 & 69.000 \\
Paulinus & Trier & 103.370 & 104.000 \\
Kath. Sonntagsblatt & Würzburg & 83.451 & 88.000 \\
\hline & & & \\
\hline
\end{tabular}

Die Bezugspreise ${ }^{41}$ der Bistumszeitungen sind, trotz Anhebungen in den letzten Jah ren, durchweg sehr niedrig. Am teuersten ist die Kirchenzeitung Köln mit 4,95 DN pro Monat, gefolgt von „Der Pilger” in Speyer mit 4,20 DM und „Konradsblatt” Frei burg mit 4,25 DM. Die meisten Bistumsblätter kosten monatlich zwischen 2,- unc 3,- DM, am billigsten ist das Passauer „Bistumsblatt” mit 1,70 DM pro Monat.

Längst sind die Bistumszeitungen - zwei verfügen über Rheinisches Format, die üb rigen halbes Zeitungsformat - auch graphisch lebendiger geworden, verwenden of auf den Textseiten Farbe, und das Foto kommt nicht zu kurz. Im Kupfertiefdrucl werden drei Bistumszeitungen hergestellt, zwei im Offsetverfahren, die restlichen in Buchdruck. Der Seitenumfang der normalen Wochenausgaben beläuft sich auf $1 t$ bis 32 Seiten. Ermittelt man die Durchschnittszahl, so ergibt sich ein Umfang vor 26 Seiten. Rechnet man dies auf ein Jahr ( 52 Wochen) um, so ergibt sich pro Bistums blatt ein durchschnittlicher Seitenumfang von 1.352 Seiten, für alle Bistumsblätter (22 zusammen 29.744 Seiten. Treibt man das Rechenexempel noch weiter und multipli ziert man das Seitenangebot aller Bistumsblätter mal knapp 2 Millionen Gesamtauf lage, so ergibt sich eine Seitenzahl von knapp 60.000.000.000. 
Will jemand alle deutschen Bistumszeitungen erwerben, so muß er dafür im Monat rund 76,- DM aufwenden, im Jahr 912,- DM. Bei einer Verkaufsauflage von 1,9 Millionen Exemplaren wiederum ergibt dies 1.800.000.000 DM (rund gerechnet). Daraus wird deutlich, daß die Bistumspresse auch einen erheblichen wirtschaftlichen Faktor darstellt. Dieser Faktor wird im Blick auf die Verlage zu wenig beachtet, wenn die Forderung nach der Einheitskirchen-Zeitung laut wird.

Denn von den 22 Bistumszeitungen erscheinen drei in einem Verlag (Fulda, Limburg, Mainz), und von den 20 Verlagen verfügen nur neun über eine eigene Druckerei. Das heißt, die meisten Bistumsblätter erscheinen im Lohndruck (und werden vor allem über ein eigenes Botennetz vertrieben). Wollte man also nur noch eine gemeinsame Zeitung für alle Bistümer herausgeben, so wären zahlreiche Verlage oder Druckereien emsthaft in ihrer Existenz bedroht. Die unterschiedlichen Formate, die dann angeglichen werden müßten, gleiches gilt für die unterschiedlichen Satztypen, und weitere Umstellungsarbeiten würden allein, so schätzen Experten, über 50 Millionen DM kosten.

Aus wirtschafts-politischer Verantwortung verbietet sich schon eine Einheitszeitung. Redaktionell jedoch vor allem. Wenn Hanisch ${ }^{42}$ eine solche Mantelausgabe fordert, dann übersieht er, daß die bisherigen 20 selbständigen Redaktionen (sozusagen Publizistische Einheiten) zugunsten einer verschwinden würden. Nun hätte das nicht unbedingt zur Folge, daß die über 100 hauptamtlichen Redakteure an Bistumszeitungen - wegen der Regional- und Lokalausgaben, die ja weiter bestehen bleiben sollen arbeitslos würden. Doch die Folgen wären unübersehbar:

- Die katholische Kirche stellt auch in der Bundesrepublik Deutschland keine Einheit dar, sondern ist diözesan vielfältig ausgeprägt. Diese Eigenheiten, die nicht unwichtig für die Leser-Blatt-Bindung sind, würden nivelliert und können durch Diözesanbeilagen nicht ausgeglichen werden.

- Die theologische Breite der Bistumsblätter wird automatisch geringer.

- Es wird schwerer, journalistischen Nachwuchs zu gewinnen. Dieser kann nur in Vollredaktionen wirklich gut ausgebildet werden.

- Ein Blick auf die Pressekonzentration in der säkularen Presse muß alle diejenigen zur Vorsicht mahnen, die mit einer Bistumszeitung für die gesamte Bundesrepublik Deutschland liebäugeln. Denn weniger Zeitungen bedeutet eben nicht größere und bessere Produkte der verbleibenden.

- Die Bindung an das Bistum kann nur verbessert werden durch eine leistungsstarke Bistumszeitung, nicht durch eine katholische Wochenzeitung für alle 22 Diözesen.

Welche Folgerungen müssen also für die Stärkung der Bistumspresse gezogen werden? Dazu einige - keineswegs vollständige - Anregungen:

- Ausbau der Redaktionen, die gegenwärtig fast alle personell unterbesetzt sind. Dies aber ist nur dann möglich, wenn der Nachwuchs von kirchlichen Journalisten systematisch gefördert wird (gute Ansätze durch die Mediendienstleistungsgesellschaft und das Institut zur Förderung publizistischen Nachwuchses in München sind vorhanden) und das schlechte Image der Kirchenpresse unter den Journalisten beseitigt wird, so daß immer mehr qualifizierte Journalisten zumindest für eine gewisse Zeit als Redakteur für eine Bistumszeitung gewonnen werden können.

- Mehr Informationen und Berichte über das tatsächliche Leben im Bistum, also weiterer Abbau der hochwürdigen Presse und Hinwendung zum Journalismus, der die Kirche widerspiegelt, wie sie tatsächlich ist. Das heißt auch, daß die Bistumspresse 
aktueller wird und mehr Informationen aus Kirche und Gesellschaft bringt, die der Leser noch nicht aus Tageszeitung oder dem Fernsehen erfahren hat.

- Ausbau der regionalen und lokalen Berichterstattung - analog der säkularen Presse, die die Bedeutung eben dieses Bereichs inzwischen längst erkannt hat.

- Ohne die älteren Leser vor den Kopf zu stoßen, müssen die Leser der mittleren und jüngeren Generation angesprochen werden. Eine Kinderseite reicht dazu nicht aus. Soziale und persönliche Fragen sind in der Berichterstattung mehr als bislang zu berücksichtigen. Die Kirchenzeitung des Bistums Münster hat beispielsweise durch die Rubrik „Der Bischof antwortet” große Resonanz gefunden - und zwar antwortet der Bischof jeweils auf eine konkrete Frage aus der Leserschaft.

- Ausbau des Nachrichtendienstes - mit Hilfe der Katholischen Nachrichtenagentu (KNA), was zwischenzeitlich auch vorgesehen ist.

- Mehr Präsenz der Bistumszeitungen in den „Presseschauen” der Tageszeitungen, am Kiosk, im gemeindlichen Leben der Pfarreien und kirchlichen Werke/Verbände.

- Stärkere Werbung durch die Pfarrer, denen im Blick auf die Verbreitung der Kirchenzeitung nach wie vor eine entscheidende Rolle zufällt. Viele Pfarrer jedoch stehen auch heute noch skeptisch der Kirchenpresse gegenüber. Diese Skepsis muß abgebaut werden.

- Der Pfarrbrief darf der Bistumszeitung keine Konkurrenz machen, sondern er muß sich auf die Berichterstattung über die Ereignisse unter dem je eigenen Kirchturm beschränken. Pfarrbrief und Kirchenzeitung ergänzen sich. Zu den Aufgaben des Pfarrbriefes gehört es auch, Werbung für den Bezug der Kirchenzeitung zu treiben.

- Obwohl die überwiegende Zahl der Bistumsblatt-Bezieher besonders kirchlich engagiert ist, dürfen die Fernstehenden in der Kirche nicht weiter unberücksichtigt bleiben. Durch besondere Themen ist diese Gruppe besonders anzusprechen, denn auch das Bistumsblatt hat eine vorrangige pastorale Aufgabe, die sich aber nicht auf die Aktiven beschränken darf.

- Förderung von Themen, die in der gegenwärtigen Massenkommunikation zu kurz kommen: Grundwertediskussion, Entwicklungshilfe, Lebenshilfe, Orientierungshilfen.

- Engere Zusammenarbeit mit der säkularen Tages- und Wochenpresse, Gastkommentare, Ausbau des Leser-Forums, Abbau des „kirchenamtlichen Nachrichtenstils”.

- Aufbau eines leistungsfähigen Vertriebsnetzes (auch das plant die Mediendienstleistungsgesellchaft), das zugleich auch der Aktualität zugute kommt, weil dadurch zum Teil der Drucktermin hinausgeschoben werden kann, ohne eine rechtzeitige Zustellung zu gefährden.

\section{Die Pressestelle - das unbekannte Wesen}

Für die Kommunikationsarbeit der Diözesen kommt den Pressestellen eine zunehmende Bedeutung $\mathrm{zu}$, die jedoch bislang keineswegs auch allgemein anerkannt ist. Im Blick auf künftige Maßnahmen der katholischen Pressearbeit darf die Differenzierung „nach den Kommunikationsräumen, in denen die Kirche ihre Kommunikationsrolle auszufüllen hat", nicht vernachlässigt werden ${ }^{42}$. Im Gegenteil. Ist der Bistumspresse „die Wir-Bildung in der Kirche anvertraut" ${ }^{43}$, so der Pressestelle der Kontakt vor allem mit den säkularen Massenmedien Zeitung, Rundfunk, Fernsehen. 
Jedes Bistum verfügt heute über mehr oder weniger gut ausgebaute Pressestellen, die oft auch eigene Information sdienste herausgeben, beispielsweise den „ndm” (Nachrichtendienst Münster) oder PEK (Pressedienst der Erzdiözese Köln). Die Schwierigkeit dieser Stellen nun liegt darin, daß sie mit ihren Informationen in Konkurrenz treten zu anderen Informationsquellen der Politik, der Wirtschaft, der Interessengruppen. Klaus Merten hat Ereignisse mit einem hohen Nachrichtenwert als „Ereignisse mit hohem Überraschungswert" ${ }^{44}$ definiert, die sich als solche dann bei den Kommunikatoren (Nachrichtenredaktionen, Redakteuren der säkularen Medien) auch durchsetzen.

Die Frage istjedoch, ob Pressestellen solche, Ereignisse mit hohem Überraschungswert” anzubieten haben. Der hohe Überraschungswert darf nicht verwechselt werden mitknalligen Sensationen, wie sie die Boulevardpresse sucht, sondern er beinhaltet im Blick auf die kirchliche Pressearbeit eine schnelle Information über wichtige Ereignisse in der Diözese, bedeutende Entscheidungen des Bistums, neue Trends in der Kirche und eine offene, nicht verwischende Information über Tatsachen.

$\mathrm{Da}$ auch für die deutsche Presse weithin der angelsächsische Spruch gilt "news is what newspapermen make it", kommt es darauf an, daß die Pressestellen der Bistümer den guten Kontakt zu den Journalisten suchen und ihre Arbeitsweise auch akzeptieren. Für die Pressestellen besteht freilich nach wie vor die größte Schwierigkeit in der Rückkopplung zum Bistum, das sich oft genug in der Presse falsch verstanden oder wiedergegeben fühlt. In Verkennung der journalistischen Wirklichkeit, daß Nachrichten stets „eine außergewöhnliche Welt” erzeugen, „da der Normalfall nicht zur Selektion zugelassen ist" 45 .

Dieser komplizierte Prozeß, der sich ja in der Regel nicht als offener Konflikt abspielt, sondern als subtiler Reibungsverlust zur Resignation führt, hat zur Folge, da $B$ die Pressestellen der Bistümer für die säkulare Presse zu unbekannten Wesen geworden sind. Nicht wenige Pressestellenleiter ziehen es deshalb vor, eng mit KNA, Bistumszeitung und einigen gut bekannten Journalisten zusammenzuarbeiten. Was fehlt, ist eine Offensivstrategie der Pressestellen auf dem Nachrichten "markt”.

Immerhin lassen sich die Diözesen die Presse- und Öffentlichkeitsarbeit längst etwas kosten. Das Erzbistum Paderborn beispielsweise gibt 1978 laut Etat 875.000 DM für Presse- und Öffentlichkeitsarbeit aus. Die Pressestelle ist mit vierhauptamtlichen Mitarbeitern besetzt. Und der Leiter der Pressestelle, Hermann-Joseph Rick, antwortet auf die Frage, ob die gegenwärtige Pressearbeit ausreiche, mit einem klaren Ja ${ }^{46}$.

Das Bistum Trier wendet für die Presse- und Öffentlichkeitsarbeit 1978484.000 DM auf. Dort wird erklärt, die Pressearbeit in den einzelnen kirchlichen Einrichtungen müsse verstärkt werden, vor allem im Blick auf die Caritas. Auch vermißt man eine „gezielte PR-Arbeit"47.

Nimmt man einen Mittelwert von 500.000 DM an, die eine Diözese pro Jahr für ihre Presse- und Öffentlichkeitsarbeit aufwendet, so ergibt sich für alle Bistümer eine Gesamtsumme von 11 Millionen DM. Hinzu kommen noch rund 15 Millionen DM, die zentral von der Medienstelle „vergeben” werden, also mindestens 26 Millionen DM.

Als beispielhaft kann die Pressearbeit des Bistums Münster gelten, die seit 1951 von Günter Graf aufgebaut wird und eine Art publizistisches Gesamtkonzept darstellt, das sich nicht nur bewährt hat, sondern auch der Nachahmung empfohlen werden kann, obwohl nur drei hauptamtliche Kräfte zur Verfügung stehen: der Leiter, ein Journalist und eine Sekretärin: 
- Die Pressestelle gibt den regelmäßigen „Nachrichtendienst Münster” (ndm) heraus, der in Zusammenarbeit mit den Tageszeitungen im Bereich des Bistums konzipiert wurde und seit vielen Jahren ,in Verbindung mit KNA" erscheint, doch nach wie vor eine eigenständige Leistung der Pressestelle darstellt. Der im 28. Jahrgang erscheinende ndm bringt viele Informationen aus dem Bistum, die sehr regional, ja'lokal abgestimmt sind. Hinzu kommt, daß wichtige Nachrichten per Fernschreiben aktuell an alle oder nur ganz wenige, weil nur regional bestimmt Redaktionen weitergegeben werden. Folge: Das monatliche Abdruckergebnis liegt bei 8.000 Druckzeilen. Obwohl das Abdruckhonorar noch nie erhöht worden ist, trägt sich dieser Dienst selbst.

- Ausbau der Bistumszeitung „Kirche und Leben” (6 Redakteure) unter besonderer Berücksichtigung der regionalen Belange. Durch breitangelegte Aufklärungsarbeit unter den Pfarrern des Bistums gelang es, das Interesse vor Ort zu stärken. Dieses wird wachgehalten durch eine intensive regionale/lokale Berichterstattung, die ihren Ausdruck in 116 Wechselseiten für die insgesamt 69 Dekanate des Bistums findet. Die Regionalseiten für die fünf Regionen des Bistums mit 2,25 Millionen Mitgliedern sollen noch weiter ausgebaut werden.

- Förderung der Pfarrbriefarbeit unter klarer Trennung zur Bistumszeitung. Die monatliche Gesamtauflage der Pfarrbriefe ist nicht festzustellen, doch rechnet Günter Graf miteiner Auflage von insgesamt 50.000 Exemplaren pro Ausgabe. Im Bistum Münster spielen die wöchentlich erscheinenden Kirchzettel (Vorläufer des Pfarrbriefs) eine erheblich größere Rolle: Gesamtauflage pro Woche 700.000 Exemplare(!).

- Weiter erscheinen im Bistum Münster als regelmäßige Publikationen „Unsere Seelsorge” (3.750 Exemplare pro Ausgabe), das „Forum” (3.150 Exemplare), „Das Familienheim" (12.000 Exemplare) ${ }^{48}$.

Noch immer werden nicht alle Pressestellen von hauptamtlichen und ausgebildeten Journalisten geleitet. In einigen wenigen nehmen diese Aufgabe Priester nebenamtlich wahr, in Osnabrück und Speyer werden die Pressestellen von Redakteuren der Bistumszeitung mit betreut. Daraus ergibt sich die Forderung, daß auch diese Pressestellen in hauptamtliche umgewandelt werden müssen, denn die Anforderungen, die die Pressearbeit beute an die Kirche stellt, können nicht nebenamtlich erfüllt werden - so qualifiziert die einzelnen Personen auch sein mögen.

Noch wichtiger aber ist die Forderung, daß die katholische Kirche auf dermittleren Ebene hauptamtliche Pressestellen einrichtet (für Regionen, Großstädte, Landkreise), deren Aufgabe nicht nur in der Nachrichtenarbeit gegenüber den Regional- und Lokalzeitungen besteht (einschließlich der Wahrnehmung der Informationsarbeit für die Verbände, Werke, kirchlichen Einrichtungen im betreffenden Gebiet), sondern auch in der Ausund Weiterbildung von ehrenamtlichen Pfarrbriefredakteuren (bzw. dem Aufbau von Pfarrbriefen, wo noch keine bestehen) sowie in der Beratung der Pfarreien und Verbände für eine gute und wirksame Öffentlichkeitsarbeit.

Diese Beratung schließt die Gewinnung ehrenamtlicher Presse- und Öffentlichkeitsreferenten ein, die für die jeweiligen Pfarrgemeinderäte oder örtlichen Verbände arbeiten. Es versteht sich von selbst, daß nicht jede Pfarrei, jedes Dekanat oder jeder Verband einen hauptamtlichen Pressereferenten anstellen kann. Das ist auch nicht erforderlich, um eine wirkungsvolle Pressearbeit leisten zu können. Hier genügt es vollständig, wenn ein hauptamtlicher Pressereferent für die mittlere Ebene auf regionaler Basis vorhanden ist. 
Ein wichtiger Bestandteil der katholischen Presse der Gegenwart ist die Ordens- und Missionspresse, deren Auflage sich gegenwärtig auf über 2,6 Millionen Exemplare beläuft und damit die auflagenstärkste Gruppe innerhalb der unterschiedlichen Gruppen katholischer Presseerzeugnisse darstellt ${ }^{49}$.

Das aber bedeutet nicht, daß die Orden auch über eine wirksame Informationspolitik gegenüber der säkularen Öffentichkeit verfügen. In den Massenmedien wird nur selten über die Orden berichtet, von einer kontinuierlichen Nachrichtenarbeit kann überhaupt nicht gesprochen werden. Hier wird in den Orden noch manche Arbeit notwendig sein, um das öffentliche Informationsdefizit abzubauen. Inzwischen „schicken” einige Orden auch Mitglieder in die Presse, vor allem Bistumszeitungen, vereinzelt sind sie auch als Redakteure bei säkularen Medien tätig. Das Frankfurter „Institut der Orden” bietet im Rahmen seiner Fortbildungsprogramme $a b$ und zu auch Seminare für praktischen Journalismus an, um den Patres und Nonnen das notwendige Handwerkszeug zu vermitteln. Diese Arbeit aber stellt erst einen Anfang dar.

Die Ordens- und Missionspresse, die bei der AKP Mitglied ist, hat vor allem zwei Zielrichtungen: Informationen über den Orden für Freunde und Förderer sowie zur Förderung der Mission, wobei den ausgesprochenen Missionszeitschriften die größte Bedeutung zukommt.

Gestaltung und Umfang dieser Zeitschriften sind sehr unterschiedlich, oft haben sie Vierfarbendruck. Reportagen und Berichte aus der Mission prägen den Inhalt dieser Blätter.

\begin{tabular}{lllr}
\hline Titel & Erscheinungsort & Erscheinungsweise & Auflage \\
\hline & & & \\
Bruder Franz & Fulda & monatlich & 11.000 \\
Das Zeichen & Limburg & monatlich & 134.000 \\
Der Rufer & Leutesdorf & monatlich & 3.000 \\
Der Weinberg & Mainz & monatlich & 92.000 \\
Die Kath. Missionen & Freiburg & zweimonatlich & 50.000 \\
Hiltruper Monatshefte & Münster & monatlich & 20.000 \\
Hoffnung & Leutesdorf & 14täglich & 90.000 \\
Kath. Apostolat & Friedberg & monatlich & 50.000 \\
Kontinente & Essen & zweimonatlich & 149.000 \\
Krankenbrief & Leutesdorf & monatlich & 51.000 \\
Mariannhill & Würzburg & monatlich & 51.000 \\
Maria siegt & Leutesdorf & monatlich & 31.000 \\
Maria vom Guten Rat & Würzburg & monatlich & 25.000 \\
Mission aktuell & Aachen & zweimonatlich & 783.000 \\
Mission aktuell & München & zweimonatlich & 270.000 \\
Pallottis Werk & Limburg & vierteljährlich & 60.000 \\
Missio Pastoral & Aachen & vierteljährlich & 14.000 \\
Schule und Mission & Aachen & vierteljährlich & 8.000 \\
Salesianische Nachrichten & München & monatlich & 67.000 \\
Sendbote & Betzdorf & monatlich & 62.000 \\
Stadt Gottes & Nettetal & monatlich & 605.000 \\
VM - Zur Zeit & Bonn & zweimonatlich & 20.000 \\
& & & \\
\hline & & & $S$ \\
\hline
\end{tabular}


In letzter Zeit hat sich die Ordens- und Missionspresse in ihrer Auflagenentwicklung ziemlich stabil gezeigt. Dies liegt nicht zuletzt daran, daß sich ein relativ fester Bezieherstamm herausgebildet hat.

Enthalten sind in dieser Übersicht nicht die vielen kleinen Publikationen der Orden und Missionen, die zum Teil von einzelnen Ordensprovinzen oder einzelnen Klöstern herausgegeben werden, beispielsweise die „Briefe der Redemptoristen”. Wie hoch die Zahl dieser Publikationen ist, läßt sich nicht ermitteln, deshalb auch nicht die Auflage, die jedoch einige zehntausend Exemplare ausmachen dürfte.

Welche Größenordnungen in finanzieller Hinsicht einige Werke der Kirche einnehmen, mag das Beispiel der Aktion „Misereor” deutlich machen, die 1976 für Öffentlichkeitsarbeit und Werbung 2,198 Millionen DM aufwandte, rund 200.000 DM mehr als im Jahr davor ${ }^{50}$.

\section{Die Zukunft kehrt zurück}

Die eigentliche Krise der katholischen Presse ist gegenwärtig überwunden. Die Anstrengungen der 70er Jahre zahlen sich aus und die Verantwortlichen können ebenso wie die Journalisten mit Hoffnung in die Zukunft schauen, die für die katholische Presse zurückgekehrt ist. Das zeigen auch die Tendenzen der übrigen katholischen Pressegruppen wie Magazine, Sonntagsblätter, Jugendzeitschriften, denen freilich der bundesweite Durchbruch bislang nicht geglückt ist. Allerdings sind noch große Anstrengungen erforderlich, um den Auflagenstand zu halten, auszubauen und neue Wege zu beschreiten, die der katholischen Presse innerhalb der deutschen Medienlandschaft den Platz sichern, der ihr zukommt.

$$
\text { - Teil II folgt - }
$$

\section{Anmerkungen}

1 Harald E. Gersfeld: Bestandsaufnahme, „Publik-Forum” 7: 1978, Nr. 18, S. 4.

2 Vgl. Hans Wagner: Das Ende der katholischen Presse, 3 Bde, Aschaffenburg 1974 (Reihe "Der Christ in der. Welt" - Eine Enzyklopädie).

${ }^{3}$ Guy Montag: Eine hoffnunsvolle Bilanz wider die Mär von der Subventionspresse, „Rheinischer Merkur" Nr. 42/1978 (20. Oktober), S. 13.

${ }^{4}$ Nach Angaben von Bruno Geuter, Geschäftsführer der AKP, gegenüber dem Vf. wurden während des 85 . Deutschen Katholikentages 1978 rund fünf Tonnen Werbeprospekte und Probeexemplare der katholischen Presse verteilt.

5 Hans Maier in der Jubiläumsausgabe der „Deutschen Tagespost” zum 30jährigen Bestehen am 28. August 1978, S. 26.

6 Guy Montag: a.a.O. Außerdem persönliche Auskünfte gegenüber Vf. durch den AKP-Vorsitzenden Ferdinand Oertel und den Leiter der Zentralstelle Medien der katholischen Kirche, Prälat Wilhelm Schätzler.

7 Verteilt an Journalisten während des 85. Deutschen Katholikentages 1978.

${ }^{8}$ Geäußert in einem Gespräch mit dem Vf. im Frühjahr 1978.

9 Heinz Wilhelm Brockmann: Nochmals: Die Weichen sind falsch gestellt, „Publik-Forum” 7: 1978, Nr. 17 (25. August) S. 22.

10 Ders.: Die Weichen sind falsch gestellt. Das einseitige Medienkonzept der katholischen Bischöfe, „Publik-Forum" 7: 1978, Nr. 13 (30. Juni), S. 3.

11 Günter Graf: Die katholische Presse in der Gegenwart, 30 Jahre Deutsche Tagespost: a.a.O., S. 25. In der gleichen Ausgabe, S. 23, beziffert Wilhelm Blank die Zahl der Blätter „katholischer Weltanschauung" vor 1848 auf 6 . Sie stieg bis 1865 auf 20 Blätter mit 60.000 Abonnenten an. 120 katholische Tageszeitungen gab es 1873 und fünf Jahre später 271. 
12 Günter Graf: a.a.O.

13 Nach Angaben der AKP.

1430 Jahre Deutsche Tagespost: a.a.O., S. 24.

is Ebd. S. 19.

16 Heinz Wilhelm Brockmann: Nochmals: Die Weichen sind gestellt, a.a.O.

17 In Zusammenarbeit mit der Mediendienstleistungsgesellchaft.

18 Wilhelm Schätzler gegenüber Vf.

19 Brockmann: Die Weichen sind falsch gestellt, a.a.O.

${ }^{20}$ „Publik-Forum” 7: 1978, Nr. 16 (11. August), S. 22.

${ }^{21}$ Herbert PrauB: Katholische Kosmetik über alten Falten, „Vorwärts” Nr. 14 (6. April 1978), S. 31.

22 Gegenüber dem Vf. in einem Gespräch. Vgl. dazu auch K. Rüdiger Durth: Vorsichtige Öffnung, „Der Journalist”, 28: 1978, Heft 5, S. 20 f.

${ }^{23}$ Ferdinand Oertel vor Volontären der Bistumspresse im Jahuar 1978 in Augsburg.

24 Ders.: Informationsverhalten und -erwartungen der Katholiken, „Herder-Korrespondenz”, Februar 1976.

2s Angaben der AKP.

26 Der Text ist dokumentiert und kommentiert von Hans Wagner in: Nachkonziliare Dokumentation, Bd. 11, Trier 1971.

${ }^{27}$ Feldbefragung. Kommunikations- und Informationserwartungen der katholischen Bevölkerung gegenüber kirchlichen Massenmedien, insbesondere der Kirchenpresse. Herausgegeben vom Institut für Kommunikationsforschung, Wuppertal 1975. Da über die Feldbefragung bereits sehr viel veröffentlicht worden ist, wird sie in dieser Abhandlung nicht näher erläutert.

28 Joseph Kardinal Höffner: Mittler zwischen Kirche und Welt, „Kirchenzeitung für das Erzbistum Köln” Nr. 48/1977, S. 9.

29 Ders.: 30 Jahre Deutsche Tagespost, a.a.O. S. 17.

${ }^{30}$ Mitteilungen der AKP, Nr. 5/1977, S. 11 f.

${ }^{31}$ Ebd. ausführlich dazu Bruno Geuter: Die Leser der konfessionellen Presse in der BRD, Informationen der AKP, 24. Juni 1972.

32 MDG-Presse-Empfang 24. April 1978 in München, Presseunterlagen.

33 Ebd.

34 Ebd.

3s Raimund Brehm: Welche Zukunft hat die katholische Presse? 30 Jahre Deutsche Tagespost, a.a.O., S. 27.

36 Gegenüber dem Vf.

${ }^{37}$ Vervielfältigter Beschlußtext.

38 Vervielfältigtes Manuskript des Deutschlandfunks.

39 Vervielfältigter Beschlußtext der Frühjahrsvollversammlung 1978.

40 Hajo Goertz: Ein Spiegel der Kirche von Köln - Überlegungen zur Aufgabe einer Bistumszeitung, „Kirchenzeitung für das ERzbistum Köln”, Nr. 33/1978, S. 3.

41 Stand 1978 nach Angaben der AKP.

42 Hans Wagner: Das Ende der katholischen Presse, a.a.O., Bd. 3, S. 58.

${ }^{43}$ Georg Moser: Die pastorale Bedeutung der Kirchenpresse, CS 7: 1974, S. 13.

4 Klaus Merten: Aktualität und Publizistik, „Publizistik” 18: 1973, S. 224.

45 Klaus Merten: Nachrichtenrezeption als komplexer Kommunikationsprozeß. Ein Beitrag zur Theorie der Nachricht, „Publizistik” 22: 1977, S. 454.

46 Angaben gegenüber Vf. vom Herbst 1978.

47 Angaben gegenüber Vf. vom Herbst 1978.

48 Angaben gegenüber Vf. in einem persönlichen Gespräch. 


\title{
SUMMARY
}

In the past decade the Catholic Church in Germany has made every effort to solve the crisis of the Catholic Press. After the closure of the weekly "Publik", Catholic Media planners directed their attention to the Diocesan weeklies and publications such as "Rheinischer Merkur" (weekly) and "Weltbild" (bi-monthly), and to the strengthening of the Catholic Nes Agency (KNA) and improving training. This was necessary on account of the decrease in circulation and the gap between the media and the Church. One of the most important developments in Catholic Communications work has been the establishment of a Media Service Company (Medien-Dienstleistungsgesellschaft - MDG) in Munich in 1975. This was part of the communications emergency planning at the Synod of the German Dioceses in 1972. The association of the Catholic Press AKP - also helped towards a revival. New and improved cooperation among the diocesan weeklies - important instruments of dialogue within the Church - was developed. The author, in describing these developments, does not confine himself to publications, but also refers to press officers of the diocese and to publications from religious communities and missionary societies which have an important role in the press today. He feels that the crisis of the Catholic Press has been overcome, but that a strenuous effort is needed to implant this same press firmly into the German secular communications media.

(Part II of this contribution will be published in a later edition.)

\begin{abstract}
RÉSUMÉ
Au cours de la dernière décennie, l'Eglise catholique fit des efforts considérables pour la presse catholique en crise. Après la suppression de l'hebdommadaire «Publik», la politique de mass media de l'Eglise consécra au renforcement des journaux épiscopaux, à la préservation du «Rheinischer Merkur» et du "Weltbild", de même que d'autres journaux et objets d'édition, au développement de l'agence de presse catholique (KNA) de même que l'encouragement de la relève journalistique. Ceci fut nécessaire parce que l'attachement à l'Eglise et avec lui le nombre d'exemplaires baissaient. Parmi les principales nouveautés sur le marché des mass media catholiques on compte aussi la société de services des mass media (MDG) - fondée en 1975 - à Munich comme partie du «programme publicistique espress» et qui fut décidée en 1972 lors du synode commun des évêchés allemands. La communauté de travail de la presse catholique (AKP) fait aussi partie des aides à l'intérieur de la publicistique catholique. A la suite d'efforts s'établit une collaboration plus étroite à l'intérieur des journaux épiscopaux qui, depuis des années, sont en train de changer fondamentalement. Ils prennent une part importante au dialogue à l'intérieur de l'Eglise. L'auteur expose les developpements et ambitions avec le conflit d'opinions. En cela, il ne se limite pas seulement aux journaux épiscopaux, mais il aborde aussi les bureaux de presse des évêchés, de même que la presse des ordres et des missions qui est également une partie intégrante importante de la presse catholique actuelle. Il est persuadé que la crise proprement dite de la presse catholique est actuellement surmontée. De gros efforts sont assurément encore nécessaires pour assurerà la presse catholique la place qui lui revient à l'intérieur du paysage des mass media allemands.
\end{abstract}

(L'exposition détaillée est poursuivie dans une deuxième partie.) 


\section{RESUMEN}

En el último decenio la Iglesia católica alemana hizo notables esfuerzos en pro de la prensa católica en crisis. Después de la supresión del semanario "Publik», la política eclesial para medios de comunicación trató de robustecer la prensa diocesana, de asegurar al periódico "Rheinischer Merkur" y a la revista "Weltbild", asi como a otras revistas y editoras, ampliando al mismo tiempo la Agencia Noticiosa Católica (KNA) y promocionando las vocaciones periodísticas. Todo ello fué necesario porque disminuyó el vínculo eclesial y con ello el número de suscriptores. Entre las más importantes novedades en el mercado de los medios de comunicación católicos se encuentra la fundación en 1975 de la MDG, una empresa de servicios para la comunicación social creada en Munich en el marco del «Programa Publicístico de Urgencia», aprobado en 1972 por el Sínodo Conjunto de las Diócesis Alemanas. También el Equipo de trabajo Prensa Católica (AKP) es uno de los puntales del publicismo católico en Alemania. Como consecuencia de estos esfuerzos surgió una estrecha colaboración entre los periódicos diocesanos, que se encuentran en evolución desde hace años. Esta prensa participa de forma importante en el diálogo interno eclesial. El autor presenta el desarrollo y aspiraciones, junto con el antagonismo de la opiniones. No se limita a la prensa diocesana: se ocupa también de las oficinas de prensa episcopales, así como de la prensa misionera y delas órdenes religiosas, que es parte importante de la prensa católica actual. Está convencido de que fué superada ya la crisis de la prensa católica. Sin embargo son necesarios todavía grandes esfuerzos para asegurar a la prensa católica el puesto que le corresponde en el panorama publicístico alemán. (La exhaustiva exposición continuará en una segunda parte.) 